\title{
Lactobacillus Rhamnosus Sepsis in an Immunosuppressed Patient with Tuberculous Meningitis and Myeloradiculitis
}

\author{
Sandra Xerinda ${ }^{1 *}$, Pedro Silva ${ }^{2}$, Carina Reis ${ }^{3}$, Antonio Sarmento ${ }^{1}$ and Lurdes Santos ${ }^{1}$ \\ ${ }^{1}$ Department of Infectious Disease-Nephrology Research an Development Unit (FCT-725), Faculty of Medicine, University of Porto, Portugal \\ ${ }^{2}$ Department of Neurosurgery, Faculty of Medicine, University of Porto, Portugal
}

${ }^{3}$ Radiology Department - Faculty of Medicine, University of Porto. C. H. São João. Porto - Portugal

\begin{abstract}
Portugal has reduced the incidence of tuberculosis disease in last years, but has an incidence that surpasses the average in Europe. The risk of reactivation of latent tuberculosis in patients about to undergo immunosuppressive and immunomodulatory therapy is high, and therefore, screening and treatment is mandatory in order to avoid the evolution of the disease from latent to active, with severe forms often difficult to treat.

We report a case of a 37 years old female, previously medicated with prednisolone and mycophenolate mofetil for a bullous pemphigus until the diagnosis of disseminated tuberculosis. In the course of the disease, a central venous catheter was placed for total parenteral nutrition due suspicion of poor absorption of antituberculous drugs. The patient had an endocarditis and septic thrombophlebitis with bacteremia due to Lactobacillus rhamnosus and difficult to treat tuberculosis complicated with meningomyeloradiculitis, which resulted in paraplegia associated with a neurogenic bladder.
\end{abstract}

\section{Introduction}

Despite the advances of microbiology, immunology and pharmacology, tuberculosis (TB) remains a serious public health problem, particularly in poor countries. Portugal, which has reduced the incidence of the disease in 2009, had an incidence of new cases of about 27.0 per 100,000 inhabitants, a figure which surpasses the average in Europe (15/100,000 inhabitants) and also values considered of low endemicity ( $<20$ cases/100,000 inhabitants) [1]. In patients about to undergo immunosuppressive and immunomodulatory therapy, the risk of reactivation of latent tuberculosis is high and therefore, screening is mandatory in order to avoid the evolution of the disease from latent to active, with severe forms often difficult to treat.

The extra-pulmonary involvement of the central nervous system (CNS) occurs in approximately $1 \%$ of cases. CNS TB is the most severe form of the disease with high morbidity and mortality (20\% to $50 \%)$, and is often associated with serious complications and permanent sequelae [2].

\section{Case Report}

A 37-year-old Portuguese female patient was hospitalized in February 2011 in another hospital unit with the diagnosis of disseminated tuberculosis (meningeal and pulmonary). As relevant past history, the patient had been diagnosed with bullous pemphigus 2 years before, and had been medicated, until the date of admission, with a prednisolone and mycophenolate mofetil.

The diagnosis of tuberculosis was based on clinical symptoms and microbiologic study: presence of acid-fast bacilli (AFB) in gastric aspirate and PCR for Mycobacterium tuberculosis in cerebrospinal fluid. She started antituberculous therapy with isoniazid (INH), rifampicin (RIF), pyrazinamide (PZA) and ethambutol (EMB). Serum testing for HIV was negative. On the day 22 of hospitalization, diplopia and paresis of the sixth cranial nerve appeared. She had fever, bad general condition with progressive malnutrition (weight $=39 \mathrm{~kg}$ ). The brain CT showed no changes. Cerebrospinal fluid (CSF) study showed white blood cell count $76 / \mathrm{mm}^{3}$ (72\% lymphocytes), protein $252 \mathrm{mg} / \mathrm{dL}$, and glucose $35 \mathrm{mg} / \mathrm{dL}$ and $\mathrm{ADA}=192 \mathrm{U} / \mathrm{L}$. In CSF culture grew Mycobacterium tuberculosis, which was susceptible to INH, RIF, PZA and EMB.
In day 50 of hospitalization, due to suspicion of malabsorption, a central venous catheter (CVC) was placed in the right subclavian vein for parenteral nutrition and parental antituberculosis drugs. The patient showed significant improvement.

On the day 66 of hospitalization, CVC nosocomial sepsis was diagnosed. Blood cultures were collected and empirically antibiotics were started. Trans-thoracic echocardiogram showed a large echogenic mass in the right atrium, mobile, protruding into the right ventricle in diastole; the mass appeared to have been originated in the superior vein cava (SVC), suggesting a thrombus or intra-cavity vegetation. The diagnosis of septic thrombophlebitis and infective endocarditis was considered.

The patient was transferred to our Hospital on the day 69 of hospitalization and admitted to the Infectious Disease Ward. She was clinically and hemodynamically stable without signs of peripheral embolization. Antituberculous therapy was restarted orally with $\mathrm{INH}+\mathrm{RIF}+\mathrm{PZA}+\mathrm{EBM}$. Given the clinical stability, the thoracic surgery was programmed to the $2^{\text {nd }}$ of May, but no thrombus was found. In postoperative period, she was admitted in the Intensive Care Unit of Infectious Diseases (ICU-ID). The evaluation in the postoperative period showed a pulmonary thromboembolism (PTE) and a thrombus of the SVC in CT angiography. Given the severity of PTE, it was decided to initiate fibrinolytic therapy.

Fever and persistently positive blood cultures were documented between days 66 and 71. Cultural identification of the microorganism

*Corresponding author: Sandra Xerinda, Department of Infectious Disease, Faculty of Medicine, University of Porto, Portugal, Tel: +351 225512 100; Fax: +351 225512216; E-mail: sxerinda@hotmail.com

Received April 21, 2013; Accepted May 05, 2013; Published May 07, 2013

Citation: Xerinda S, Silva P, Reis C, Sarmento A, Santos L (2013) Lactobacillus Rhamnosus Sepsis in an Immunosuppressed Patient with Tuberculous Meningitis and Myeloradiculitis. J Trop Dis 1: 107. doi:10.4172/2329-891X.1000107

Copyright: $\odot 2013$ Xerinda S, et al. This is an open-access article distributed unde the terms of the Creative Commons Attribution License, which permits unrestricted use, distribution, and reproduction in any medium, provided the original author and source are credited. 


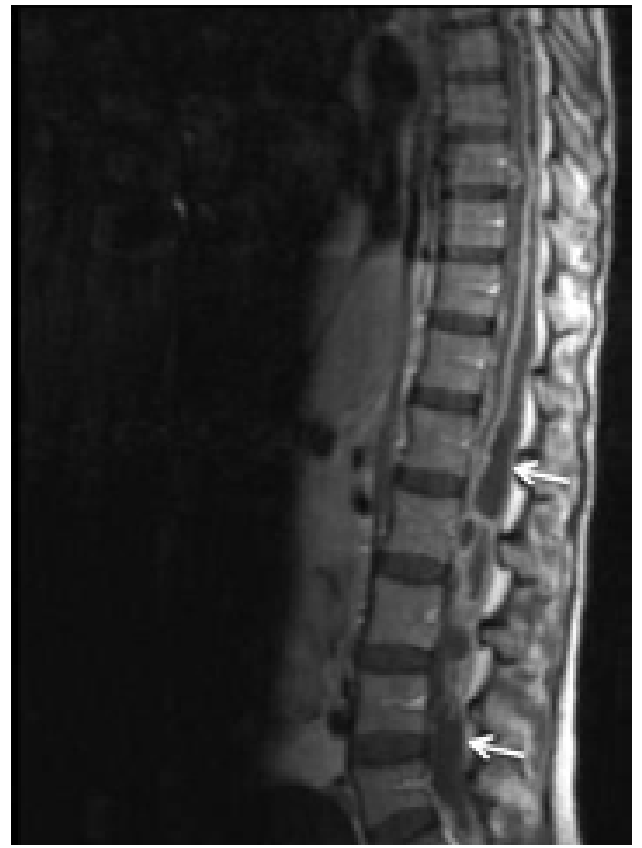

Figure 1: The brain and spinal cord MR

had been difficult but, with the help of molecular biology, a Lactobacillus rhamnosus was identified in blood cultures. Antimicrobial Susceptibility Test (AST) was obtained by E Test. She made multiple antibiotic regimens, the last one with ampicillin, clarithromycin and amikacin according to AST. With the latter, and due to the interaction of clarithromycin with rifampicin, it was decided to swap rifampicin with ciprofloxacin. There was a progressive deffervescence, reduction of inflammatory parameters and blood cultures became negative on the day 16 of antibiotic therapy based on AST. The patient improved and was discharged from ICU-ID. She completed 6 weeks of antibiotic therapy for Lactobacillus rhamnosus bacteremia.

On day 121 of antituberculous treatment, she developed progressive lower limb weakness with difficulty in walking and bladder disturbance. Neurological examination showed paraplegia with a level of sensitivity in the right D6 and D8 in the left lower limb with arreflexia and neurogenic bladder. The brain and spinal cord MRI (Figure 1) showed intracanalar collection interesecting the medullary subdural space T4-L4; abscesses in intramedullary topography of the vertebral body at the level of $\mathrm{T} 1$ and sub-pial at $\mathrm{C} 3$, with edema surrounding spinal cord, were observed, and these changes were compatible with spinal empyema and abscesses. The patient underwent laminectomies of D5D11, and L4 levels with drainage of purulent material. The ZN showed the presence of $\mathrm{AFB}$ and it was decided to reboot PZA and EMB, which had already been suspended, associated with INH, RIF, ciprofloxacin and meropenem. Methylprednisolone $(1 \mathrm{mg} / \mathrm{Kg} /$ day $)$ was added to the therapeutic regimen.

The spinal MRI performed four weeks after surgery showed persistence of a large area of hyperintensity on T2, extending from D1 to the medullary cone, compatible with medullar suffering. The patient was, once again, submitted to D3-L5 laminectomy for drainage of empyema. The $\mathrm{ZN}$ and cultures of the exudates were negative for mycobacteria.

The patient progressed favourably and the spinal MRI performed after the second surgery showed smaller sub-pial abscesses collection, compared to the previous MRI.

The patient remains under treatment for tuberculosis and has had a partial recovery of sensory and motor deficits. She is still in a physical rehabilitation programme.

\section{Discussion}

Mycophenolate mofetil is an immunosuppressor with antilymphocytic effect used in renal transplant and autoimmune diseases and is associated, among other complications, with some risk of opportunistic infections, including tuberculosis. Strategies to reduce the risk of tuberculosis imply the diagnosis of latent tuberculosis and its treatment, particularly in patients who will be submitted to immunosuppressive drugs [3].

The case under study shows two unusual complications: bacteremia caused by Lactobacillus rhamnosus and a severe outcome of tuberculosis meningitis, despite adequate treatment. The Lactobacillus rhamnosus infection is sporadically seen in patients with impaired host defenses and severe underlying diseases, as well as prior surgeries, but infection of endovascular surfaces is uncommon [4]. Lactobacillus bacteremia range from asymptomatic to severe sepsis and may be combined with pneumonia, deep abdominal abscesses, or endocarditis, a rare focalization [5]

Lactobacilli may be difficult to cultivate, and conventional identification methods reveal only $30 \%-50 \%$ of the isolates [6]. In the case reported, it was not possible to identify the infection using traditional methods, even with persistent positive blood cultures (several series), and it was only possible with the help of polymerase chain reaction (PCR). In the literature, sensitivity by the E-test method varies. Treatment should be prolonged and optimized according to the E-test results [7].

Regarding the nosocomial events, the catheter-related bacteremia, complicated by septic thrombophebitis and pulmonary thromboembolism, contributed to the persistence of positive cultures. The patient described developed a very rare and severe form of tuberculosis - a myeloradiculitis. Moreover, it has appeared after 4 months of appropriate therapy based on susceptibility testing, behaving like a paradoxical response. This paradoxical response may explain the fact that the patient, even with appropriate therapy, has progressed towards subdural empyema and intramedullary spinal cord abscess, very rare forms of neurological involvement. The diagnosis may have been delayed because the patient had been sedated and ventilated for several weeks.

The use of steroids in tuberculous of CNS is controversial. There is concern regarding the bioavailability of the antituberculous drugs entrance in CNS, upon restoration of the blood-brain barrier due to the anti-inflammatory effects of corticoids. However, there is evidence to suggest that their use is warranted and beneficial $[8,9]$. In our case, steroids were not started initially when meningitis was diagnosed because mental status was preserved and no neurologic deficit was present. The tuberculosis myeloradiculitis can occur by three ways: as a primary tuberculous lesion, as an extension upstream of tuberculous meningitis, or as a secondary extension of spinal tuberculosis. Macroscopically, a common feature is the presence of extensive exudates [8].

A combination of antituberculousis drugs should be initiated soon after diagnosis and treatment, with or without surgical 
Citation: Xerinda S, Silva P, Reis C, Sarmento A, Santos L (2013) Lactobacillus Rhamnosus Sepsis in an Immunosuppressed Patient with Tuberculous Meningitis and Myeloradiculitis. J Trop Dis 1: 107. doi: 10.4172/2329-891X.1000107

Page 3 of 3

intervention, should last at least 12 months. In some situations, myeloradiculitis occurs not due to the failure of antituberculosis drugs, but to a paradoxical reaction to treatment $[10,11]$. The importance of laminectomy remains controversial; however, it should always be considered when evidence of spinal cord suffering is present, as in the case described $[5,12,13]$. The myeloradiculitis tuberculosis is rare, but its diagnosis should be considered when a patient develops symptoms of spinal cord involvement, as in the present case. MRI is the method of choice for diagnosis.

In conclusion, this case report shows two rare diagnosis: catheter associated bacteremia complicated by septic thrombophlebitis by Lactobacillus rhamnosus and a disseminated form of tuberculosis complicated by myeloradiculitis during treatment.

\section{References}

1. Direcção Geral de Saúde. Programa Nacional de Luta contra a Tuberculose Março de 2010. Accessed in January 2013.

2. Liao PW, Chiang TR, Lee MC, Huang $\mathrm{CH}$ (2010) Tuberculosis with meningitis, myeloradiculitis, arachnoiditis and hydrocephalus: a case report. Acta Neurol Taiwan 19: 189-193.

3. Sayiner A, Ece T, Duman S, Yildiz A, Ozkahya M, et al. (1999) Tuberculosis in renal transplant recipients. Transplantation 68: 1268-1271.

4. Husni RN, Gordon SM, Washington JA, Longworth DL (1997) Lactobacillus bacteremia and endocarditis: review of 45 cases. Clin Infect Dis 25: 10481055.
5. Antony SJ, Stratton CW, Dummer JS (1996) Lactobacillus bacteremia: description of the clinical course in adult patients without endocarditis. Clin Infect Dis 23: 773-778.

6. Parola P, Maurin M, Alimi Y, Juhan C, Brouqui P (1998) Use of 16S rRNA gene sequencing to identify Lactobacillus casei in septicaemia secondary to a paraprosthetic enteric fistula. Eur J Clin Microbiol Infect Dis 17: 203-205.

7. Salminen MK, Rautelin H, Tynkkynen S, Poussa T, Saxelin M, et al. (2006) Lactobacillus bacteremia, species identification, and antimicrobial susceptibility of 85 blood isolates. Clin Infect Dis 42: e35-44.

8. Chotmongkol V, Kitkuandee A, Limpawattana P (2005) Tuberculous radiculomyelitis (arachnoiditis) associated with tuberculous meningitis. Southeast Asian J Trop Med Public Health 36: 722-724.

9. Freilich D, Swash M (1979) Diagnosis and management of tuberculous paraplegia with special reference to tuberculous radiculomyelitis. J Neurol Neurosurg Psychiatry 42: 12-18.10.

10. Escobar JA, Belsey MA, Dueñas A, Medina P (1975) Mortality from tuberculous meningitis reduced by steroid therapy. Pediatrics 56: 1050-1055.

11. Carvalho AC, De laco G, Saleri N, Pini A, Capone S, et al. (2006) Paradoxica reaction during tuberculosis treatment in HIV-seronegative patients. Clin Infect Dis 42: 893-895

12. Vlcek B, Burchiel KJ, Gordon T (1984) Tuberculous meningitis presenting as an obstructive myelopathy. Case report. J Neurosurg 60: 196-199.

13. Giménez-Roldán S, Esteban A, Benito C (1974) Communicating syringomyelia following cured tuberculous meningitis. J Neurol Sci 23: 185-197. 